\title{
Computational Model for Large Volume Expansion and Self-Contact with Adaptive Meshing
}

\author{
Abhiroop Satheesh*, Christoph Meier* and Wolfgang A. Wall* \\ * Institute for Computational Mechanics, Technical University of Munich, Boltzmannstr. 15, \\ 85748 Munich, Germany, e-mail: satheesh | meier | wall@lnm.mw.tum.de, \\ http://www.mw.tum.de/lnm
}

\begin{abstract}
Key Words: Finite deformation thermo-mechanics, multiplicative deformation decomposition, thermo-mechanical contact, mesh-refitting.

A variety of foam products are used in different industrial branches such as construction, where they serve e.g. as gap fillers and even for fixing parts like doors and windows instead of more complicated fixing systems. In order to improve, in the sense of handling and efficiency, those products, especially for sensitive applications, a reliable predictive simulation approach is needed. Existing models for foam expansion are almost exclusively limited to the study of 1D problems. This is not surprising as volume expansion ratios can reach values up to $1000 \%$, and multi-dimensional models require the inclusion of rheological properties of the expanding material (which is not easy to measure) and the handling of a large number of (self-) contacts with potentially complex and rapidly changing contact interface topologies. On the other hand, a robust computational model for multidimensional expansion would be highly desirable since typical applications involve complex $3 \mathrm{D}$ geometries.

In this work, the large expansion is modelled through a multiplicative decomposition of the deformation gradient into a expansion part and an elastic part, which was first introduced by Lee [1] in the context of plasticity. Building upon this multiplicative decomposition, an evolution equation in rate form is employed to account for the volumetric expansion. Since the underlying material parameters are typically temperature-dependent, a fully coupled thermo-mechanical problem is solved in a monolithic manner. For a robust numerical treatment of multi-body contact and selfcontacting scenarios involving frictional, thermo-mechanical interaction, powerful mortar methods for computational contact and interface mechanics [2] are employed. A mesh-refitting algorithms is employed to preserve the mesh under large deformations.
\end{abstract}

\section{Acknowledgements}

The authors acknowledge the financial support from the European Union's Horizon 2020 research and innovation programme under the Marie Skodowska-Curie grant agreement No 764636.

\section{REFERENCES}

[1] E. H. Lee, "Elastic-plastic deformation at finite strains," J. App. Mech., vol. 36, no. 1, pp. 1-6, 1969.

[2] A. Seitz, W. A. Wall, and A. Popp, "Nitsches method for finite deformation thermomechanical contact problems," Comp. Mech., pp. 1-20, 2018. 